\title{
THE EFFECTS OF MENSTRUAL CYCLE ON THE TASK PERFORMANCE USING A BODY WARMER FOR THE PALLIATION OF MENSTRUAL ASSOCIATED SYMPTOMS
}

\author{
Kasamatsu, K. ${ }^{1}$, Suzuki, S. ${ }^{2}$, Karashima, M. ${ }^{3}$, Kumashiro, M. ${ }^{1}$, Ninomija, P.S. ${ }^{2}$ \\ ${ }^{1}$ Department of Ergonomics, University of Occupational and Environmental Health, JAPAN \\ ${ }^{2}$ Department of Integrated Information Technology, Aoyama Gakuin University, JAPAN \\ ${ }^{3}$ Department of Management Systems Engineering, Tokai University, JAPAN \\ kkasa@med.uoeh-u.ac.jp
}

\begin{abstract}
The task performance and a questionnaire survey using the modified MDQ (Menstrual Distress Questionnaire) were examined for phases of the menstrual cycle. The lower abdomen was warmed using a body warmer to palliate menstrual associated symptoms. The effect of palliation using this folk remedy on the task performance was examined. The experimental tasks consisted of clicking on randomly displayed targets and a single tracking task. The task performance and MDQ score were measured as measurement indices. As the results, the precision of task during menstruation was lower than during the follicular phase. There were many complaints regarding menstrual associated symptoms during menstruation. The palliation treatment did not relieve the negative symptoms experienced by the subjects.
\end{abstract}

\section{INTRODUCTION}

The menstrual cycle is a unique biologically rhythmic cycle which can have various side effects on women throughout the cycle, not just during menstruation itself. These side effects are influenced by diverse factors. In addition, there are various side effects which occur when women are in the menstrual cycle itself. These effects not only differ between individuals, but also show intra-individual variations. The menstrual cycle most noticeably accounts for intra-individual variations among individuals.

Anse et al.(1994) and Kasamatsu et al.(2002, 2003) investigated the effects of the menstrual cycle on various tasks, and reported fluctuation in task performance coincident with the menstrual cycle. Dalton and Holton(1999) reported that certain mental and physical capacities change in women in relation to the menstrual cycle. Kumari and Corr(1996) suggested that the effects of the menstrual cycle, conceptualized as partly arousalbased, interact with situational manipulations of arousal to influence performance on intelligence tests. Moos (1968) created a questionnaire, called the Menstrual Distress Questionnaire (MDQ), to measure complaints and distress in relation to the menstrual cycle. The MDQ is a list of 47 symptoms divided into a scale of eight factors by factor analysis. Those eight factors are 'Pain', 'Autonomic reactions', 'Water retention', 'Concentration', 'Behavioral changes', 'Negative affect', 'Arousal', and 'Control'.

While responses to menstrual phases will vary from person to person, a large sample size may offset those differences and produce a consistent phase-related pattern. Therefore, it is necessary to examine and compare the effects of the menstrual cycle on different individuals.

The previous studies have investigated the relationship between the menstrual cycle and task performance, and menstrual complaints. In this study, the effect of palliation for menstrual associated symptoms on task performance was examined. The lower abdomen was warmed using a warmer to palliate the menstrual associated symptoms. Moreover, the MDQ by Moos was modified to be culturally specific to Japan, and menstrual complaints in Japanese women were investigated using this modified MDQ.

\section{METHODO LOGY}

Task performance and a questionnaire survey using the modified MDQ were examined for phases of the menstrual cycle. The lower abdomen was warmed using a warmer to palliate menstrual associated symptoms. The effect of palliation using this folk remedy on task performance was examined. 


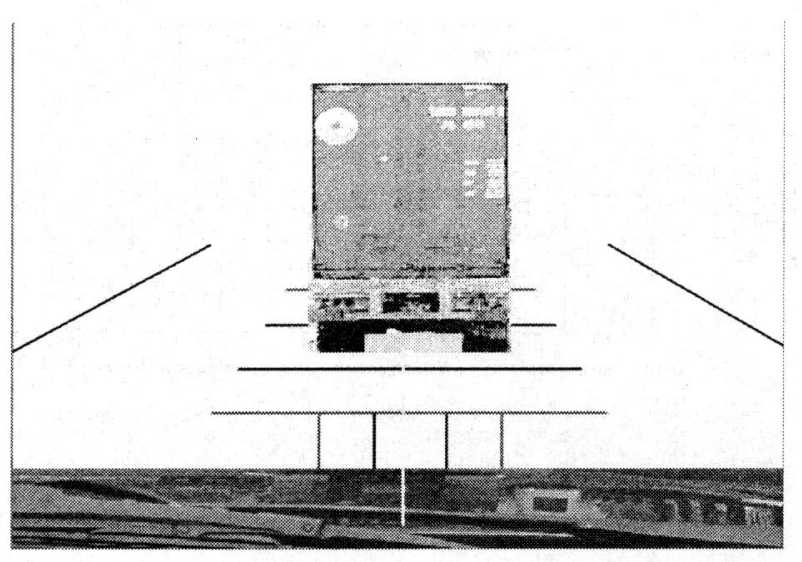

Fig. 1. The screen of a single tracking task

Subjects

The inclusion criteria for subjects in this study were as follows: unmarried women with a regular menstrual cycle, who did not take hormones or other drugs, were not pregnant, and had not habit smoking. All subjects were volunteers. To define the menstrual cycle, the basal body temperature was taken and recorded every morning. Subjects who the basal body temperature was divided into low and high temperature periods were selected, and the subjects' menstrual cycles were confirmed to be fairly regular. The 12 subjects were college students, with a mean age of 21.6.

\section{Menstrual Cycle}

Menstruation, menstruation during which the menstrual associated symptoms were palliated (the palliation phase), and the follicular phase were selected and examined. The experiment on menstruation was carried out within 48 hours after bleeding began. The experimental order of the three phases of the menstrual cycle was arranged as a counterbalance.

\section{Experimental Tasks}

The two experimental tasks were used a single tracking task, performed, and a target clicking task, which was also performed on the computer. These tasks were performed at each phase of the menstrual cycle. The experimental time was fifteen minutes on each task.

A single tracking task: A single tracking task is a kind of tracking task in which a car is modeled on a Visual Display Terminal (VDT) (Figure 1), and the subjects must maintain a fixed distance from the car, and must also endeavor to stay

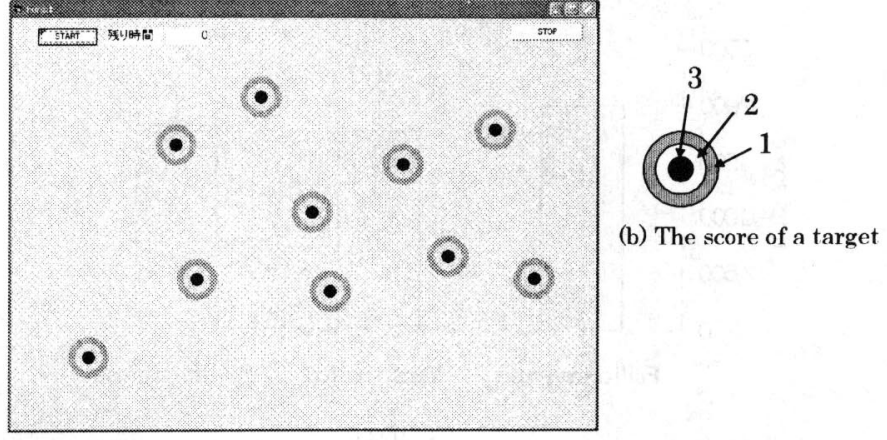

(a) The screen of a target clicking task

Fig.2. The screen of a target clicking task (a) and the score of a target (b)

in line with the car, which is displayed as being ahead of them. The subjects perform this task using four keys on the keyboard. Two levels of conditions were provided concerning the distance between the car the subject was operating and the car in front, as well as the secession from right to left. The first level required the subjects to not let the front car jut out onto the blue lines, and the second level required them to not let the front car jut out onto the red lines. Moreover, the subjects were supposed to try to keep the yellow bar on top of each other at the yellow point.

A target clicking task: A target clicking task consisted of clicking on targets which were displayed randomly, which made them disappear. This had to be accomplished with a certain time limit (Figure 2). The ten colored targets such as those shown in Figure 2(a) were displayed randomly. The following requirements were given to the subjects: Click the center of the targets as accurately as possible, and make them disappear, all within a certain time limit. The time limit given was eleven seconds. This task was the first stage, and continued for fifteen minutes. After the time limit was up, all targets disappeared, and the next stage was begun. The scores for the colored targets were 3 as the core (the inside agenda), 2 (the inside blue), and 1 as the outermost (the inside yellow)(Figure 2(b)). A time limit on each stage was displayed on the screen. The residual time of the total experimental time were not displayed.

\section{Measurement Indices}

The rate which fulfilled two level conditions was measured as the task performance in a single tracking task. The total number of targets that disappeared (the number of tasks), the score on a stage, the time which was taken to complete a stage (completed time), the total score, and the error rate were measured as the task performance in a target clicking 


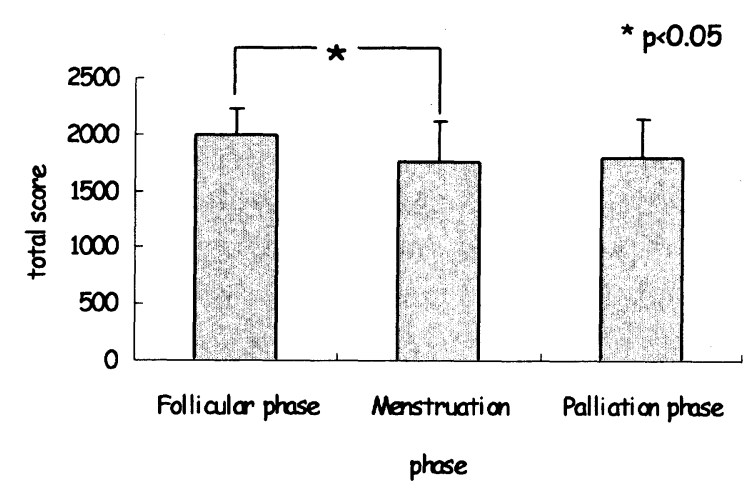

Fig. 3. The mean value of the total score on target clicking task task. Critical flicker frequency was measured both before and after the experiment was begun. Flicker value was measured five times. The mean value of three measurements, with the exception of the maximum and minimum, were calculated.

Moreover, the MDQ by Moos was modified to be culturally specific to Japan and one item was added. The modified MDQ with 48 items in Japanese was administered to investigate the physical and mental conditions of the subjects at pre- and post-experiments. The MDQ was estimated on a six-point scale, which ranged from no experience with the symptom, to significant experience.

\section{RESULTS}

\section{Task performance}

A single tracking task: The result of the rate within the blue line of both back-forth and right-left was examined using ANOVA. There is no significant difference between three phases. However, the rate within the blue line during the palliation phase was the lowest.

A target clicking task: The differences between phases were examined using ANOVA on the measured task results. As the results, there were significant differences between phases on the total score and the score on the stages $(p<0.05)$. Moreover, the multiple comparisons between phases were performed on the total score and the score on the stages. The total score (Fig. 3) and the score on the stages of the follicular phase were significantly higher than those on menstruation $(p<0.05)$. There was a significant tendency for the scores on the follicular phase to be higher than those on the palliation phase $(\mathrm{p}<0.10)$. The score on the stages was also the same result as the total score. The error rate on menstruation was lower than the palliation phase. There

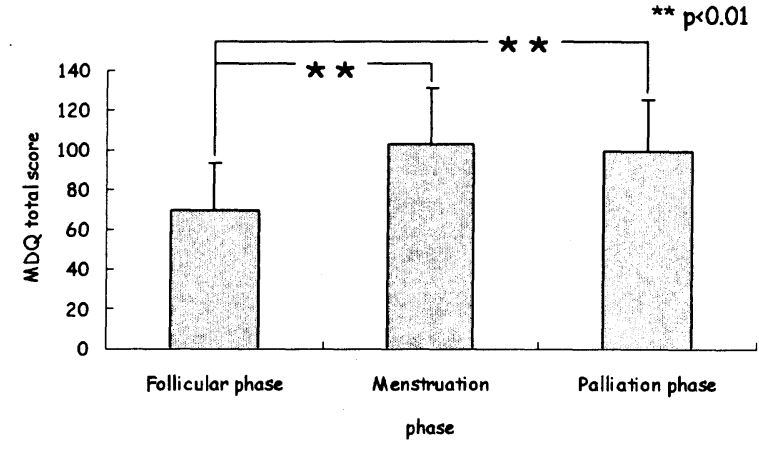

Fig. 4. The total score of MDQ on pre-experiment

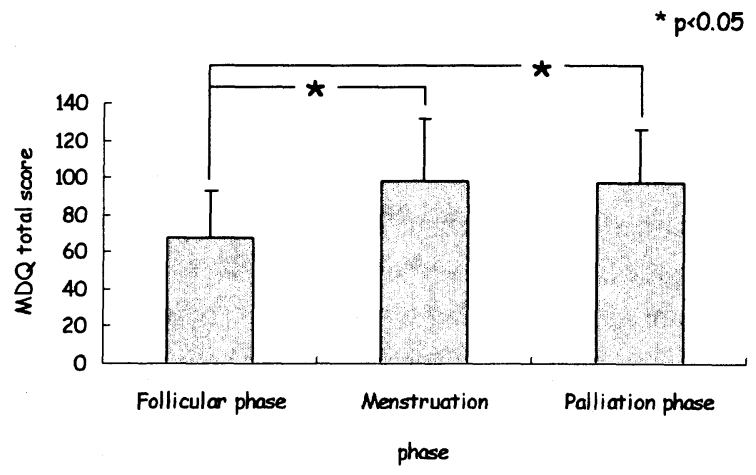

Fig. 5. The total score of MDQ on post experiment

was no significant difference between the phases on the number of tasks performed, completion time, and reaction time.

\section{Flicker Test}

The flicker value between the pre- and the post-experiment was examined using t-test. As a result, there were significant differences between the pre- and the postexperiment on all phases $(\mathrm{p}<0.05)$.

The rate of change in the flicker value each phase was examined using ANOVA. There was no significant difference between phases on the rate of change in the flicker value. However, the change of the flicker value during the palliation phase was the largest.

\section{A Modified MDQ}

The highest score phase on the mean value of each factor in the pre-experiment was as follows: the palliation phase on pain, menstruation on concentration, menstruation on behavioral changes, the palliation phase on autonomic reactions, menstruation on water retention, menstruation on 
negative affect, menstruation on arousal, and the palliation phase on control. The highest score phase in postexperiment was as follows: the palliation phase on pain, the palliation phase on concentration, menstruation on behavioral changes, the palliation phase on autonomic reactions, menstruation on water retention, menstruation on negative affect, menstruation on arousal, and menstruation and the palliation phase on control.

The difference between the three phases was examined using ANOVA on the total score and eight factors score, and each symptom score on the pre- and postexperiments. ANOVA results on the pre-experiment were as follows (Fig.4). For the total score, there were significant differences between the three phases $(p<0.01)$. The results of multiple comparison showed that the total score of the menstruation $(p<0.01)$ and palliation phase $(p<0.01)$ were significantly higher than that of the follicular phase. ANOVA results on the post-experiment were as follows (Fig.5). There was a significant difference between the three phases on the total score $(p<0.05)$. The results of multiple comparison showed that the total score of the menstruation $(p<0.05)$ and palliation phase $(p<0.05)$ were significantly higher than that of the follicular phase. There was no significant difference between the menstruation and palliation phase.

\section{DISCUSSION}

From examining task performance, it was found that the score during the follicular phase was significantly higher than the score during menstruation, and the score during the follicular phase tended to be higher than the score during the palliation phase. That is, there was a higher precision of task during the follicular phase. However, there was no significant difference between the menstruation and palliation phases, and it was suggested that there was no effect of palliation on task performance. The concentration factor and lowered judgment received higher scores postexperiment on the palliation phase. The subjects became especially fatigued during the palliation phase.

The total score during the menstruation and palliation phase was higher than the follicular phase on the preexperiment MDQ. The scores for pain, behavioral changes, and water retention during the menstruation and palliation phase were significantly higher than for the follicular phase, and there was a strong tendency for the subjects to experience physical complaints during the menstruation and palliation phase.
It was suggested that warming the lower abdomen was connected to relax of mental condition and palliation of pain. While there was condition which can expect an effect during palliation, the condition causing the negative effect that lowered judgment appeared. However, the effect of palliation was not recognized on task performance. It was suggested that palliation did not have an effect on mental tasks, such as the experimental tasks performed in this study, and that a palliative effect cannot be expected, because there was no significant difference between the menstruation and palliation phase on task performance, and deterioration in judgment was indicated.

\section{CONCLUSION}

The precision of task during menstruation was lower than during the follicular phase. There were many complaints regarding menstrual associated symptoms during menstruation. While there was condition which can expect an effect during palliation, the condition causing the negative effect that lowered judgment appeared.

\section{REFERENCES}

Anse M., Kasamatsu K., Funada F. M., Idogawa K., and Ninomija P.S. 1994. Effect of menstrual cycle on the stability of the task performance in the monotonous work, Proc. of 16th Annual Int Conf of the IEEE-EMBS, 311-312. Dalton K., and Holton W. 1999. Once a month Understanding and treating PMS-. 6th ed. Alameda, Hunter House Inc.

Kasamatsu K., Suzuki, S. Anse M., Funada F. M., Idogawa K., Ninomija P.S. 2002. Menstrual cycle effects on performance of mental arithmetic task, Journal of Physiological anthropology and applied human science, 21(6), 285-290.

Kasamatsu K., Kumashiro M., Izumi H., Karashima M., Suzuki S., and Ninomija P.S. 2003. The menstrual cycle effects on the computer-paced work, The 10th Annual Meeting \& Conference of Ergonomics Society of Taiwan, 57-62.

Kumari V., and Corr P. J. 1996. Menstrual cycle, arousalinduction, and intelligence test performance, Psychol Rep, 78, 51-58.

Moos R. H. 1968. The development of a menstrual distress questionnaire, Psychosom Med, 30(3), 853-867. 